Portland State University

PDXScholar

7-6-2021

\title{
Incarceration and Suicide: Do the Risk Factors Differ for Civilians and Veterans?
}

Rheannon Gail Ramsey

Portland State University

Follow this and additional works at: https://pdxscholar.library.pdx.edu/open_access_etds

Part of the Criminology Commons, Mental and Social Health Commons, and the Military and Veterans Studies Commons

Let us know how access to this document benefits you.

\section{Recommended Citation}

Ramsey, Rheannon Gail, "Incarceration and Suicide: Do the Risk Factors Differ for Civilians and Veterans?" (2021). Dissertations and Theses. Paper 5715.

https://doi.org/10.15760/etd.7588

This Thesis is brought to you for free and open access. It has been accepted for inclusion in Dissertations and Theses by an authorized administrator of PDXScholar. Please contact us if we can make this document more accessible: pdxscholar@pdx.edu. 
Incarceration and Suicide: Do the Risk Factors Differ for Civilians and Veterans?

by

Rheannon Gail Ramsey

A thesis submitted in partial fulfillment of the requirements for the degree of

Master of Science

in

Criminology and Criminal Justice

Thesis Committee:

Christopher Campbell, Chair

Brian Renauer

Mari Matsuda

Portland State University

2021 


\begin{abstract}
Suicide is one of the leading causes of death in United States jails and prisons. Many researchers have looked at suicides in prisons and what can potentially cause suicidal ideation but there are conflicting findings among civilian incarcerated populations and United States military veteran incarcerated populations.

The intent of this study is to examine which risk factors are most prevalent among adults in custody, with a focus on mental health and substance use or abuse and how these risk factors differ between incarcerated civilians and incarcerated veterans. Using survey data from the 2002 Survey of Inmates in Local Jails conducted by the Bureau of Justice Statistics and running a logistic regression on three models, a "top five" ranking of risk factors was found for each sub-population.

I found that diagnosed mental health factors were the largest indicators of suicidal ideation within the sample. Veterans differed slightly from civilians in which mental health disorders predicted a higher likelihood of suicidal ideation with other mental condition being the top risk factor for veterans and depressive disorder being the top risk factor for civilians. There was an interesting finding among race that spanned all three models showing adults in custody who self-identified as Native American, Alaskan Native, or Hawaiian/Pacific Islander were at greater risk of suicidal ideation.
\end{abstract}




\section{Table of Contents}

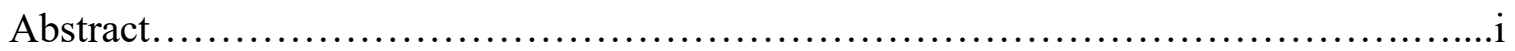

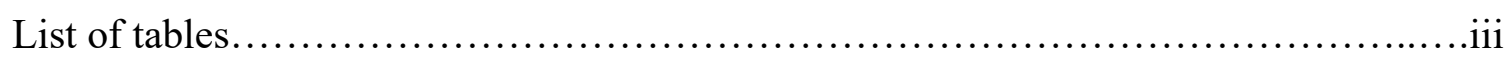

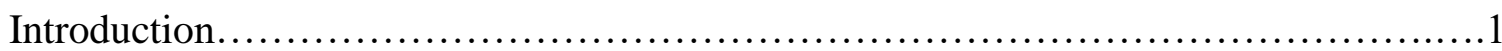

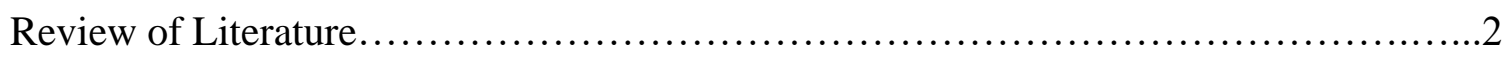

Nonincarcerated Civilian Population and Suicide............................2

Nonincarcerated Veteran Population and Suicide..............................

Civilian Incarcerated Population and Suicide.................................5

Veteran Incarcerated Population and Suicide...............................6

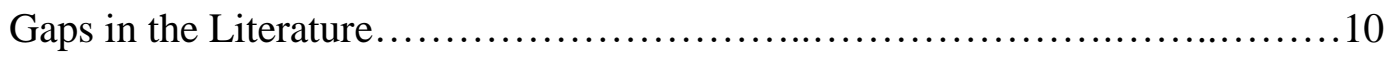

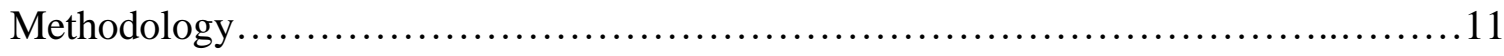

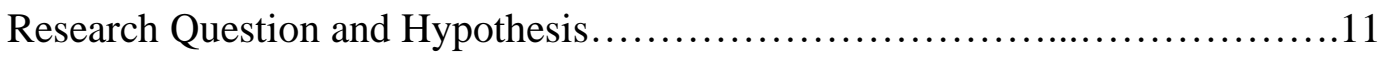

Participants and Original Data.........................................11

Measures......................................................... 12

Analytic Plan........................................................ 14

Results.................................................................14

Discussion............................................................. 16

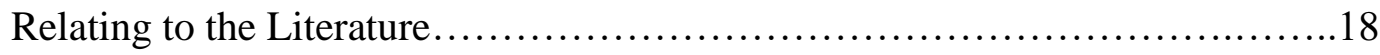

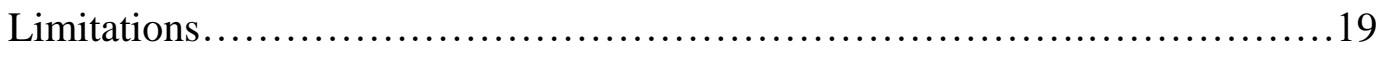

Policy Implications ..............................................20

Conclusion...............................................................21

References...........................................................22 


\section{List of Tables}

Table 1: Sample descriptives................................................... 13

Table 2: Logistic regressions predicting suicidal thoughts or attempts by sub-populations 15

Table 3: “Top 5" risk factors for sub-populations.....................................16 


\section{Introduction}

Suicide is the leading cause of death in United States jails and second leading cause in prisons (Chui, 2018). In 2014 the suicide rates were 50 per 100,000 jail inmates, 20 per 100,000 state prisoners, and 14 per 100,000 federal prisoners. Suicide rates were higher for incarcerated individuals than nonincarcerated individuals with 1,896 incarcerated suicides (Dixon et al., 2020). By identifying factors that are more likely to lead to suicide for each population, we may be more likely to help these populations and reduce the number of incarcerated suicides. Among those incarcerated, there is a special population who sits at the crosshairs of two risk factors prone to suicide: Incarcerated veterans (Wortzel, 2009). Among the most thought of risk factors that could lead a person to suicide, mental health is perhaps the most influential. In this context, there are many issues related to mental health to consider; post-traumatic stress disorder (PTSD), anxiety, depression, bipolar disorder, and schizophrenia are a just a few. There are also factors such as drug and alcohol use or abuse that are common ushers for suicide.

Studies on veterans in jails and prisons are important because they can lead to reforms in the United States Department of Veterans Affairs (VA) and how they treat out-processing veterans and which services they offer and to whom they offer them. The VA does not offer services to all outcoming veterans, usually just those who have been rated with service-related disabilities and have honorable or general with honorable condition discharges, and many veterans do not know how to get VA benefits (U.S. Department of Veterans Affairs, 2021). Not having these resources can hinder veterans' ability to transition to civilian life. 
The central aim of this study is to examine the intersection of veteran status, mental health, and drug use among those incarcerated. Ideally looking for a "top five" kind of ranking of risk factors for suicidal ideation among these populations. Specifically, I compare which factors are associated with an increased risk of suicide for incarcerated civilians, and which factors may lead to an increased risk for incarcerated veterans. The primary research question this thesis addresses is: To what degree do veterans and nonveterans differ in their risk factors for suicide? Using the 2002 Survey of Local Inmates data collected by the Bureau of Justice Statistics, I analyze indicators of mental health, substance and/or drug use, and other factors that may be predictors of suicide for the incarcerated civilian and incarcerated veteran populations. For this study, suicidal ideation is defined as considering or attempting suicide.

\section{Literature Review}

\section{Nonincarcerated Civilian Population and Suicide}

A study conducted by Han, et al. using the 2008-2011 U.S. Mortality Files and the 2008-2012 National Surveys on Drug Use and Health investigated fatal and nonfatal suicide cases. They highlighted that in 2012, 1.3 million adults aged 18 and over reported attempting suicide in the past twelve months. Using their data, Han and colleagues found that 39,426 adults died by suicide and another 2,000 adults reported attempting suicide. Using descriptive analysis and a multivariate logistic regression, they found the top risk factors for suicide were being 45 years or older, white, male, and having less than a high school education (Han, et al. 2016). 
Noting an increase in suicide cases in the United States, Olsen et al. conducted a study using the 2004-2005 Wave 2 National Epidemiologic Survey on Alcohol and Related Conditions (NESARC) and the 2012-2013 NESARC-III which are nationally representative self-report surveys containing identical questions. The initial collection efforts asked 69,321 participants aged 21 years or older about the occurrence of suicide attempts within the last three years. After adjusting for race, sex, and race/ethnicity they found that the participants with the most recent suicide attempts were females under the age of 50 years old. Other factors included being between 21-34 years of age, having a high school education or less, a history of violent behavior, and being diagnosed with antisocial personality disorder, anxiety, or depressive disorder (Olsen, et al. 2017).

Steele, et al. conducted a systematic review of literature using multiple databases of studies conducted within the last twenty years in the United States with 100 or more participants and found static and dynamic risk factors for suicide in adults. Adults ages 20-64 years of age were at a greater risk of suicide if they were white males with past or current substance abuse issues and recent job losses or financial difficulties. This study found that in 2014 suicide rates had reached a high point from the last 25 years with 42,773 reported in the United States in that year. Other risk factors for suicide included personal history of non-suicidal self-injury (NSSI), psychiatric disorders, military service, and less than high school education (Steele, et al. 2018).

\section{Nonincarcerated Veteran Population and Suicide}

In a report by Bossarte, et al they note that there was a $60 \%$ increase in suicides reported by the Veterans Health Administration in 2012. Factors such as service history, 
mental health, and time since separation can all impact suicidal ideation in veterans. Using data from the 2010 Behavioral Risk Factor Surveillance System (BRFSS) and a questionnaire administered by the Veteran's Health Module (VHM) administered in two states, Nebraska and Tennessee, these researchers aimed to find which risk factors lead to suicidal ideation among veterans. Out of 2,602 participants 3.8\% reported having suicidal ideation and $0.4 \%$ reported attempting suicide in the previous twelve months. This data showed that older, non-Hispanic veterans who had been diagnosed with a psychiatric disorder were the most likely to report suicidal ideation. Depressive disorder, anxiety, and post-traumatic stress disorder (PTSD) were among the top indicators (Bossarte, et al. 2012)

In a study conducted by Wiener and colleagues, they looked at veteran morality following a previous suicide attempt using a retrospective cohort study. The participants received inpatient care during the years of 1993 through 1998 and various Veterans Affairs (VA) medical facilities across the United States. The recorded deaths happened between the years 1992 to 2002 and were identified through the VA Beneficiary and Records Locator System data and the National Death Index Data. This study found that during 1993 though 1998, 10,163 veterans had been treated and discharged by a VA medical center following a suicide attempt. There were high amounts of diagnosed alcohol and drug dependence or abuse rates in these veterans. Psychoses, depression, and hypertension also were highly diagnosed. 1,836 veterans had died during the follow-up period, making about $78 \%$ survival rate. The leading causes of death for the veterans in 
this study were $20.2 \%$ heart disease, $13.2 \%$ suicide, and $12.7 \%$ unintentional injury (Weiner, et al. 2011).

A study conducted by Wood, et al in 2020 compared the risk factors for suicide in male veterans and nonveteran males. In a sample size of 21,692 male veterans and 83,430 nonveteran males using data from the National Violent Death Reporting System, they found that the veteran population had a smaller number of factors but in higher rates, the biggest one being physical health problems. For veterans, they were more likely to leave a suicide note, recently experienced the death of a close friend or family member, and have physical health problems.

\section{Civilian Incarcerated Population and Suicide}

In United States' jails suicide has been noted as being the leading cause of death for adults in custody (Chui, 2018). For prisons, this is all too similar with suicide being the second leading cause of death. These shocking statistics, she claims are due to the custodial environment adults in custody face and also the adult in custody's difficulty coping with the prison or jail environment. Many, if not most, correctional facilities generally but especially in jails lack the staff knowledge and comprehensive suicide prevention policies to help reduce or eliminate inmate suicides. Between 2013 and 2014, the suicide rate rose over $13 \%$. Chui states that in order to help eliminate incarcerated suicides, the federal government should allocate funding to state and local facilities for suicide prevention training and protocols (Chui, 2018).

A study conducted by Schaefer, et al. (2016) on suicidal ideation mentions that suicidal ideation is a significant indicator of potential suicide attempts. In a mixed- 
gender, jail sample consisting of 511 participants, they found that $16 \%$ of adults in custody reported having suicidal ideation. They found that suicidal ideation was greatest upon incarceration and higher for those adults in custody who were Caucasian, had a history of previous suicide attempts, and some kind of psychiatric diagnosis. This study found no difference in sex or age when it came to the prevalence of suicidal ideation (Schaefer, et al. 2016).

Dixon and colleagues (2020) looked into suicides committed while in jail or prison. Using data from the National Violent Death Reporting System (NVRDS) 20032014, which is a Centers for Disease Control and Prevention (CDC) state-based active surveillance system that collects and tracks detailed information on all violent deaths in the United States including suicides, they tested for risk factors of suicides completed during incarceration. Dixon, et al. (2020) used chi-squared tests for demographics, the characteristics of the incident, and the circumstances of the suicide and a logistic regression to decipher significant results. They found that those who committed suicide while in jail or prison had higher rates of substance abuse, had been incarcerated for person crimes, and were most likely to commit suicide by hanging themselves with bedding or clothes. Incarcerated individuals were more likely to commit suicide when between the ages of 25 to 39 years old, belonging to a racial or ethnic minority group, and unmarried (Dixon, et al. 2020).

\section{Veteran Incarcerated Population and Suicide}

In a special report examining demographics, military service, disability status, and mental health status of incarcerated veterans, Bronson et al. (2015) used data form the 
2011-12 National Inmate Survey (NIS-3). The survey captured information from adults in custody in 233 state and federal prisons, 358 local jails, and 15 special facilities including military, Indian county, and Immigration and Customs Enforcement (ICE) facilities. Overall, the survey was administered to 41,822 respondents in state and federal prisons and 58,745 in jails, making a total of 100,567 incarcerated adult participants. There was a small number of female participants making it impossible to make reliable inferences about female veterans, so females were excluded from all analyses. The potential source of bias the authors found was nonresponse. Responses were weighted for them to be able to produce national-level estimates. Significant findings form this report include the fact that in spite of an incarceration rate for veterans being lower than that for nonveterans (a steady decline over 20 years), veterans were more likely to be sentenced for violent offenses, $77 \%$ of incarcerated veterans had honorable or under honorable conditions discharges from the military, and combat veterans were more likely than noncombat veterans to have been diagnosed with a mental disorder (Bronson, et al. 2015).

Many veterans report having psycho-social problems while also facing diverse levels of criminality and criminogenic needs. Schaffer (2016) notes that transitioning from military life to civilian life is challenging for many veterans and have varying coping techniques. There is a population of veterans who isolate, turn to substance abuse and alcoholism, domestic violence, assaults, or have untreated psycho-social issues that may cause them to enter the criminal justice system. Schaffer states "the number of incarcerated veterans are likely to increase, especially within the Persian Gulf War and Operation Enduring Freedom/ Operation Iraqi Freedom populace” (p. 303). While much 
of this is due to the high volume of veterans involved in these conflicts, it may also be due to the moral waiver system that came along with the unpopularity of the Persian Gulf War. The moral waiver process allowed for entering servicemen and women to have criminal backgrounds including misdemeanors and some felony offenses. This moral waiver populace became Operation Enduring Freedom/ Operation Iraqi Freedom recruits, and this prior criminality could play a factor in veterans being involved within the criminal justice system. Schaffer states that veteran treatment courts can help unburden the courts, Veteran's Affairs, and military systems while also helping veterans thrive outside of the criminal justice system by providing medical, psychiatric, housing, and other resources needed to help veterans cope and not become incarcerated (Shaffer, 2016).

Diving further into the intersection of mental health and incarceration among veterans, White et al (2012) conducted a study to examine the prevalence and causes of military veterans in the arrestee population. In their study, the authors mention that mental health issues date back as far as the Civil War with literature speaking of "nervous disease" faced by soldiers in war. "Nervous disease" is described closely to what we now call PTSD. Looking at mental health, substance abuse, and military service, the authors sought to find a potential link from military service to being incarcerated. This study was conducted using survey data coupled with data from Arizona Arrestee Reporting Information Network of 2,102 recent arrestees in Maricopa County, Arizona. The surveys were conducted with randomly selected arrestees based on their time of booking, although arrestees who had been in custody for more than 48 hours were ineligible for 
participation due to time limitations with urinalysis. Of the 2,102 respondents, 132 reported prior military service. To compare the nonveteran and veteran populations, the authors used a two-stage analytic strategy including a basic descriptive analysis and logistic regression. The authors found that veterans were predominantly male and significantly older than their nonveteran counterparts. Veterans were more likely to achieve a post high school education, be working full time at the time of arrest, be living with their spouse, be arrested for a violent offense, and more likely to report having a mental health problem. In contrast, veterans were no more likely to be homeless than nonveterans. There were several limitations involved with this study. The data was collected from just one county located in Arizona. Additionally, the authors were only able to examine incarcerated veterans, excluding civilians from the analysis. Though there were some differences between incarcerated veterans and nonveterans, the authors were unable to make a significant inference about military service and criminality being linked. They state that more long-term studies need to be conducted on mental health and substance abuse in veterans (White et al., 2012).

A meta-analysis conducted by Wortzel et al (2009) focused on incarcerated veterans and the complications involved in studying this special population. They collected their data using studies that were found using key words associated with incarcerated veterans and suicide rates. They found that much is unknown about veterans and incarcerated veterans when it comes it mental health and suicide rates. Wortzel et al states that "few outcomes represent a greater failure at reintegration into civilian life among our returning veterans than suicide" (p. 82). They point out that though this topic 
has received much attention from the media, little has been done about it in terms of policy and research. It is also stated that this is an incredibly special population that is hard to study due to how incarcerated veterans sit in the intersection of two already protected populations, prisoners and veterans. This meta-analysis highlights possible risk factors for suicide in incarcerated veterans such as race (specifically white males), violent offenses, high rates of mental illness, substance abuse, age, homelessness, and prior incarceration. The authors note that jails and prisons should collect veteran status as part of the intake process in order to be better able to study this topic. Veteran suicide is a national problem that can be attributed to failed reintegration into civilian life (Wortzel et al., 2009).

\section{Gaps in the Literature}

There are many studies about suicide, incarcerated suicide, and veteran suicide. These studies show that suicide is prevalent in all populations: civilians, veterans, and incarcerated populations. What we do not know is what the risk factors are for civilians versus what the risk factors are for veterans. There are different conclusions on male versus female, race and ethnicity, substance and alcohol use, and mental health conditions. The aim of this study is to sort those differences out.

There are mixed findings in all of the subpopulations. Olsen, et al (2017) has conflicting findings to the Han, et al (2016) findings on general risk factors such as age, high school education, and past behavior which could be areas for further research in the general non-offending population. In the civilian sub-group, there were once again mixed findings on how sex and age may affect suicidal ideation in the Shaefer, et al (2016) 
study. Dixon, et al (2020) had contrary findings that stated suicidal ideation was higher among those in minority groups, while other studies say those who identified as White have higher rates. For the veteran population, there are small numbers of female veterans in the incarcerated population which often makes females excluded from the overall studies.

\section{Methodology}

Research Question and Hypothesis

The research question this thesis seeks to address is: To what degree do veterans and non-veterans differ in their risk factors for suicide? To answer this question, I will be using a cross-sectional design by using data from a survey of incarcerated adults. I will be looking for a "top-five" type of risk factors for suicide and how they might differ between incarcerated veterans and civilians. I hypothesize that diagnosed depressive disorders will be the top risk factor for civilian suicidal ideation and diagnosed PTSD will be the top risk factor for veteran suicidal ideation. The previous literature on veteran suicide mentions PTSD as one of the most common risk factors for suicide, while depressive disorder is mentioned in much of the civilian suicide literature, which is how these hypotheses came to be used for this study.

\section{Participants and Original Data}

The data used for this secondary data analysis was originally gathered by the United States Department of Justice, Bureau of Justice Statistics for the 2002 Survey of Inmates in Local Jails. The data was collected in a stratified two-stage selection process. The first stage consisted of selecting which jails to use for the data collection while the 
second stage consisted of choosing the inmates to interview. The data was collected through a survey of defendants being held before trial and those that have been convicted and are serving sentences or waiting to be transferred to other facilities.

Overall, there were 6,982 interviews completed with 768 non-interviews, 263 adults in custody refused participation, 407 adults in custody were released after sampling by either transfer to another facility, to court, or other types of release and there were 98 adults in custody who could not be interviewed due to medical, security, or other administrative reasons (U.S. Department of Justice). Excluding cases with missing data (201 cases), for this study I will be using 6,771 participants form the original survey and running three separate models to assess my measures.

\section{Measures}

For this study I used the following variable as descriptive measures. V5: Sex-Sex Recode Variable was kept as the original coding since the variable is already a dichotomous male/female response. V14: Age (Original) was also kept in original coding, age in years. For race/ethnicity, Variables V28-33: Descriptive race was coded into the categorical race/ethnicity breakdowns of (1) White, (2) Black or African American, (3) Hispanic or Latinx, (4) Native American, Alaskan Native, or Hawaiian/ Pacific Islander, and (5) All other races/ethnicities. To create my civilian and veteran populations, I used V58: Served in the U.S Armed Forces, which was coded dichotomously into Veteran/Nonveteran.

All the mental health variables had been diagnosed by a mental health care professional. For this study the following mental health diagnoses were used; V2022: had 
a depressive disorder, V2023: had bipolar disorder, V2024: had psychotic disorder, V2025: had post-traumatic stress disorder, V2026: had other anxiety disorder, and V2028: had other mental condition. For the drug and alcohol measures V2831: regular alcohol use, V2831: regular drug use, and V2486: drug dependence or abuse were used.

The final variables being used were also recoded into dichotomous variables of “yes" and "no"; V2044: ever attempted suicide and V2046: ever considered suicide. These variables have also been combined to the variable: thought_attempt_suicide in order to test the above risk factors against self-reported suicidal ideation among the incarcerated population.

Table 1: Sample descriptives

\begin{tabular}{|c|c|c|c|}
\hline Measures & $\begin{array}{c}\text { Veterans } \\
\text { Only }\end{array}$ & $\begin{array}{c}\text { Civilians } \\
\text { Only }\end{array}$ & $p$ \\
\hline \multirow[t]{2}{*}{$N$} & 496 & 6,275 & \\
\hline & Freq $(\%)$ & Freq $(\%)$ & \\
\hline Mean age (standard deviation) & $41.5(10.0)$ & $30.7(9.9)$ & $<.001$ \\
\hline Sex $($ Male $=1)$ & $457(92.1)$ & $4394(70.0)$ & \\
\hline Race/ethnicity & & & $<.001$ \\
\hline White & $241(48.6)$ & $2237(35.6)$ & \\
\hline Black or African American & $190(38.3)$ & $2526(40.3)$ & \\
\hline Hispanic or Latinx & $28(5.6)$ & $825(13.1)$ & \\
\hline Native Amer., Alaskan Native, or HI/ Pac. Islander & $25(5.0)$ & $319(5.1)$ & \\
\hline All other races/ethnicities & $12(2.4)$ & $368(5.9)$ & \\
\hline Current offense: Violent & $141(28.4)$ & $1507(24.0)$ & .028 \\
\hline \multicolumn{4}{|l|}{ Military service branch } \\
\hline Army (includes National Guard) & $284(57.3)$ & - & - \\
\hline Navy or Coast Guard & $95(19.2)$ & - & - \\
\hline Marine Corps & $71(14.3)$ & - & - \\
\hline Air Force & $36(7.3)$ & - & - \\
\hline Other & $10(2.0)$ & - & - \\
\hline \multicolumn{4}{|l|}{ Mental health } \\
\hline Depressive Disorder & $385(77.6)$ & $4870(77.6)$ & .995 \\
\hline Bipolar Disorder & $442(89.1)$ & $5521(88.0)$ & .455 \\
\hline Psychotic Disorder & $30(6.0)$ & $284(4.5)$ & .121 \\
\hline Anxiety Disorder & $452(91.1)$ & $5679(90.5)$ & .646 \\
\hline PTSD & $442(89.1)$ & $5899(94.0)$ & $<.001$ \\
\hline Other Mental Disorder & $486(98)$. & $6135(97.8)$ & .754 \\
\hline \multicolumn{4}{|l|}{ Drug use } \\
\hline Regular Alcohol Use & $131(26.4)$ & $2395(38.2)$ & $<.001$ \\
\hline Regular Drug Use & $332(67.2)$ & $4345(69.3)$ & .325 \\
\hline Drug Dependence or Abuse & $268(54.0)$ & $2824(45.0)$ & $<.001$ \\
\hline Considered or attempted suicide & $137(27.6)$ & $1663(26.5)$ & .587 \\
\hline
\end{tabular}




\section{Analytic Plan}

I created three different models using binary logistic regression and then using the exponentiated beta coefficients (i.e., odds ratios, OR) to interpret my results and crosstabs for descriptive statistics of my sample. I separated my models by all adults in custody, veterans only, and civilians only. Model 1 includes all participants in the survey to determine the overall risk factors for the population with a veteran variable included as a potential risk factor. Model 2 includes veterans only and the variables for potential risk factors. Finally, Model 3 is the civilian only participants and the variables for potential risk factors.

\section{Results}

Table 2 provides the results of the three models used in this study. The results from the binary logistic regression shows that the mental health and substance factors had the strongest impact on whether or not an adult in custody had considered or attempted suicide. Across all models, mental health factors were in the "top five" risk factors while substance and alcohol use were secondary. Race/ethnicity showed that across all three models Native American, Alaskan Natives, Hawaiian/Pacific Islanders had greater odds of suicidal ideation than any other category.

In model one, the all adults in custody model, those diagnosed with a mental disorder possessed substantially and significantly greater odds of considering or attempting suicide than those without the noted disorder. Those with a depressive disorder had 3.1 times greater odds, those with a bipolar disorder had 2.3 times greater odds, those with a psychotic disorder had 2 times greater odds, those who had been 
diagnosed with other mental disorders had 2 times greater odds, and those diagnosed with PTSD had 1.7 times greater odds to consider or attempt suicide.

Table 2: Logistic regressions predicting suicidal thought or attempts by sub-population

\begin{tabular}{|c|c|c|c|c|c|c|}
\hline \multirow{3}{*}{ Independent measures/controls } & \multirow{2}{*}{\multicolumn{2}{|c|}{ 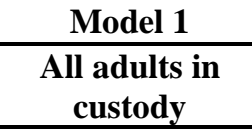 }} & \multicolumn{2}{|c|}{ Model 2} & \multicolumn{2}{|c|}{ Model 3} \\
\hline & & & \multicolumn{2}{|c|}{ Veterans only } & \multicolumn{2}{|c|}{ Civilians only } \\
\hline & OR & $p$ & OR & $p$ & OR & $p$ \\
\hline Veteran & 1.19 & .173 & - & - & - & - \\
\hline Age & 1.00 & .304 & .99 & .472 & .99 & .345 \\
\hline Sex $($ Male = 1) & .57 & $<.001$ & 1.14 & .783 & .55 & $<.001$ \\
\hline \multicolumn{7}{|l|}{ Racelethnicity (ref. $=$ White) } \\
\hline Black or African American & .63 & $<.001$ & .46 & .007 & .64 & $<.001$ \\
\hline Hispanic or Latinx & .70 & .001 & .44 & .162 & .72 & .004 \\
\hline Native Am., AK Native, HI/Pac. Isl. & .90 & .464 & 1.55 & .390 & .86 & .313 \\
\hline All other races/ethnicities & .79 & .099 & .08 & .113 & .82 & .180 \\
\hline Current offense: Violent & 1.41 & $<.001$ & .99 & .991 & 1.44 & $<.001$ \\
\hline \multicolumn{7}{|l|}{ Mental health } \\
\hline Depressive Disorder & 3.12 & $<.001$ & 5.16 & $<.001$ & 3.03 & $<.001$ \\
\hline Bipolar Disorder & 2.29 & $<.001$ & 1.21 & .665 & 2.39 & $<.001$ \\
\hline Psychotic Disorder & 2.00 & $<.001$ & 2.13 & .138 & 2.01 & $<.001$ \\
\hline Anxiety Disorder & 1.51 & $<.001$ & 3.54 & .006 & 1.44 & .001 \\
\hline PTSD & 1.67 & $<.001$ & 2.12 & .060 & 1.63 & $<.001$ \\
\hline Other Mental Disorder & 1.97 & $<.001$ & 6.75 & .032 & 1.86 & .002 \\
\hline \multicolumn{7}{|l|}{ Substance use } \\
\hline Regular Alcohol Use & 1.35 & $<.001$ & 1.37 & .299 & 1.36 & $<.001$ \\
\hline Regular Drug Use & 1.31 & .004 & 1.03 & .943 & 1.33 & .004 \\
\hline Drug Dependence or Abuse & 1.68 & $<.001$ & 1.53 & .001 & 1.63 & $<.001$ \\
\hline \multicolumn{7}{|l|}{ Model fit indices } \\
\hline Chi-square (p value) & \multicolumn{2}{|c|}{$1537.62(<.001)$} & \multicolumn{2}{|c|}{$159.55(<.001)$} & \multicolumn{2}{|c|}{$1401.83(<.001)$} \\
\hline df & \multicolumn{2}{|c|}{17} & \multicolumn{2}{|c|}{16} & \multicolumn{2}{|c|}{16} \\
\hline Nagelkerke $\mathrm{R}^{2}$ & \multicolumn{2}{|c|}{.297} & \multicolumn{2}{|c|}{.400} & \multicolumn{2}{|c|}{.293} \\
\hline$-2 \log$ likelihood & \multicolumn{2}{|c|}{6279.32} & \multicolumn{2}{|c|}{419.91} & \multicolumn{2}{|c|}{5835.48} \\
\hline
\end{tabular}

In model two, the veterans only model, those who were diagnosed with other mental disorders had 6.7 greater odds to commit or attempt suicide, those with a depressive disorder had 5.1 times greater odds, those with an anxiety disorder had 3.5 times greater odds, those with a diagnosed psychotic disorder had 2.1 greater odds and 
those diagnosed with PTSD had 2.1 times greater odds than veterans in custody those without these diagnoses.

In model three, the civilians only model, those with a diagnosed depressive order had 3 times greater odds to consider or attempt suicide, those diagnosed with bipolar disorder had almost 2.4 times greater odds, those with a diagnosed psychotic disorder had 2 times greater odds, those diagnosed with other mental disorders had 1.9 times greater odds, and those diagnosed with PTSD had 1.6 times greater odds to have considered or attempted suicide than other civilians in custody without these diagnoses.

Across all three models, the mental health variables had the highest odds ratios. Substance use was the second category with highest odds ratios. Although some variables in the analysis did not reach the level of significance, these results still suggest variables such as being a veteran have a greater percentage chance of having suicidal ideation.

\section{Discussion}

The results of the regression showed that the race/ethnicity with the greatest odds of suicidal ideation across all three models was those participants who identified as Native American, Alaskan Native, or Hawaiian/ Pacific Islander, and diagnosed mental health conditions were most indicative of suicidal ideation. We know that overall depressive disorder is the leading risk factor, when broken into groups that stays true for civilians but not for veterans. The leading risk factor for suicidal ideation in incarcerated veterans is other mental disorders, which is not specified other than being diagnosed with any other mental or emotional condition. Although veteran status was not significant in 
model one, those with veteran status had a $19 \%$ more chance of having suicidal ideation that their civilian counterparts.

Table three shows the "top five" risk factors for each model. This ranking system was pulled from table two. The five variables with the highest odds-ratios were used to determine this "top five" of the risk factors. Since the main goal of this study was determine which risk factors for suicidal ideation among the populations were more likely, odds-ratios were used instead of significance values.

Table 3: "Top 5" Risk Factors for sub-populations

\begin{tabular}{llllll}
\hline \multicolumn{1}{c}{ Adults in Custody } & \multicolumn{2}{c}{ Veterans Only } & \multicolumn{2}{c}{ Civilians Only } \\
\hline Risk Factor & OR & Risk Factor & OR & Risk Factor & OR \\
\hline 1. Depressive disorder & 3.12 & 1. Other mental condition & 6.75 & 1. Depressive disorder & 3.03 \\
2. Bipolar disorder & 2.29 & 2. Depressive disorder & 5.16 & 2. Bipolar disorder & 2.39 \\
3. Psychotic disorder & 2.00 & 3. Anxiety disorder & 3.54 & 3. Psychotic disorder & 2.01 \\
4. Other mental condition & 1.97 & 4. Psychotic disorder & 2.13 & 4. Other mental disorder & 1.86 \\
5. PTSD & 1.67 & 5. PTSD & 2.12 & 5. PTSD & 1.63 \\
\hline
\end{tabular}

My hypothesis for the civilian population was supported by these findings in that depressive disorder was found to be the top risk factor for civilians who are incarcerated. The adults in custody model and civilian model had the same ranking in risk factors and very similar odds ratios. My hypothesis for the veteran population was wrong, PTSD was not the top risk factor, it was the fifth. The other mental disorder was the number one risk factor for the incarcerated veteran population. This could be due older veterans not being diagnosed with PTSD, rather "shell shock" or other variants and PTSD could be underdiagnosed in these populations. Also worth noting is that anxiety disorder was only in the top five for the veteran population but bipolar disorder which is present in both model 1 and model 2, is not. 
There is much literature on civilian and veteran suicides, incarcerated suicides seem to be understudied. This study was focused on the incarcerated population with an emphasis on veteran status.

\section{Relating to the Literature}

The results of my tests were similar to the literature due to mental health and substance abuse being the most prominent risk factors for an adult in custody to consider or attempt suicide. The results from my logistic regression were more precise in noting which mental health factors were the most common for each group to have been diagnosed with and also have suicidal ideation. Instead of just stating mental health is a large risk factor for suicidal ideation in adults in custody, it can be said for generalizability that depressive disorders are the largest risk factor overall and for civilians who are incarcerated while other mental disorders were the leading risk factor for incarcerated veterans. This is something majority of the prior literature does not specify or look at specific mental health issues.

Contrary to the Shaefer et al. (2016) study that found those who identify as White were more likely to have suicidal ideation this study found similar to Dixon et al (2020) that a minority group was had greater odds of suicidal ideation, which was those participants who identified as Native American, Alaskan Native, or Hawaiian/ Pacific Islander. Shaefer et al (2016) also found that psychiatric diagnoses were risk factors for suicide, similar to this study.

When it came to the veteran incarcerated population, the findings of this study were similar to that of the previous literature. Bronson et al (2015), White et al (2012), 
and Wortzel et al (2009) all found that veterans who had been arrested for violent offenses, have PTSD, and mental illnesses were more likely to have suicidal ideation. Wortzel et al (2009) brought up substance abuse and alcohol as risk factors, while this study did include these factors, they were not in the "top five" rankings but still showed higher odds of suicide than incarcerated veterans without substance problems.

\section{Limitations}

Since this is a study based on secondary data, the questions asked were not solely about mental health, drug and alcohol use, or suicidal ideations. The data was collected through self-report surveys so there is a possibility that some of the questions were not answered truthfully or the participant was not paying attention to the questions being asked and just filling in answers to get the survey done. Another limitation is that there was not a variable or clarification on when the adults in custody had considered or attempted suicide, some of those who answered yes could have meant prior to incarceration or military service and it could be not related to the adults in custody being incarcerated.

Another large limitation was the lack of responses for some variables. There were other measures that would have been useful in this analysis such as home life, education attainment, past criminal history, etc. Although there were variables for some of these factors, there was too much missing data that excluded these variables from the analysis.

There was also not accessible information on attempted or completed suicides while incarcerated due to the data collection being through a survey. There is also a possibility of adults in custody having a mental health disorder and not yet being 
diagnosed. Even though the mental health factors are diagnosed by a mental health professional, they are not specified such as 'other mental disorder.' The final limitation is that the data is from 2002 which appears to be the last survey where the Bureau of Justice Statistics asked in-depth mental health questions. More current data with the mental health factors included would capture the large portion of veterans coming out of service from Operation Enduring Freedom, Operation Iraqi Freedom, and Operation New Dawn. This missing population could change these results but are unfortunately uncaptured with the Bureau of Justice Statistics no longer asking about mental health and suicide. Future research needs to be done on current data with more specific mental health questions.

\section{Policy Implications}

This study showed that over one-fourth of participants had attempted or considered suicide, while for veteran participants that percentage is $27.6 \%$ and civilian participants $26.5 \%$ had attempted or considered suicide. When about one-quarter of incarcerated adults have considered or attempted suicide and the leading risk factors are surrounding mental health or substance use, it is time to make a change and provide better or more accessible mental health and substance rehabilitation services in United States jails and prisons.

In order to make a change, I propose adding future studies involving more indepth mental health questions and follow-ups. Many jails and prisons screen for mental health at the time an adult in custody is brought in, routine screenings should be taking place as mental health status may change while incarcerated. Services need to be provided for those adults in custody that are showing signs of declined or declining 
mental health status. Psychiatrists should be available to diagnose specific mental illnesses and address necessary treatment to help prevent incarcerated suicides and suicidal ideation.

\section{Conclusion}

In conclusion, mental health issues and disorders have been proven to be top risk factors for suicidal ideation among adults in custody. Substance abuse is also a factor to consider in suicidal ideation. The risk factors do vary slightly between veteran and civilian incarcerated populations. It is imperative that mental health be monitored more closely and frequently in United States prisons and jails, not just at in-take but also regularly throughout the individual's sentence as mental health status can change and deteriorate while in prison and jail. Treatment needs to be offered accordingly and specified to the individual's needs. Suicide can be prevented and should not be the top leading causes of death for adults in custody. 


\section{References}

Bossarte, R. M., Knox, K. L., Piegari, R., Altieri, J., Kemp, J., \& Katz, I. R. (2012). Prevalence and characteristics of suicide ideation and attempts among active military and veteran participants in a national health survey. American Journal of Public Health, 102(1), 38-40.

Bronson, J., Carson, E. A., Noonan, M., \& Berzofsky, M. (2015). Veterans in prison and jail, 2011-12. Bureau of Justice Statistics. 1-21.

Chui, V. (2018). Correcting correctional suicide: Qualified immunity and the hurdles to comprehensive inmate suicide prevention. Boston College Law School, 59: 13971432.

Dixon, K. J., Ertl, A. M., Leavitt, R. A., Sheats, K. J., Fowler, K. A., \& Jack, S. P. D. (2020). Suicides among incarcerated persons in 18 U.S. states: Findings from the National Violent Death Reporting System, 2003-2014. Journal of Correctional Health Care. 26(3), 279-291.

Han, B., Kott, P. S., Hughes, A., McKeon, R., Blanco, C., \& Compton, W. M. (2016). Estimating the rates of death by suicide among adults who attempt suicide in the United States. Journal of Psychiatric Research, 77: 126-133

Olfson, M., Blanco, C., Wall, M., Shang-Min, L., Saha, T. D., Pickering, R. P., \& Grant, B. F. (2017). National trends in suicide attempts among adults in the United States. JAMA Psychiatry, 74(11), 1095-1103. 
Schaefer, K. E., Esposito-Smythers, C., \& Tangney, J. P. (2016). Suicidal ideation in a United States jail: Demographic and psychiatric correlations. The Journal of Forensic Psychiatric \& Psychology, 27(5), 698-704.

Schaffer, B. J. (2016). Incarcerated veterans outreach program. Journal of EvidenceInformed Social Work, 13: 293-304. DOI: 10.1080/23761407.2015.1006945.

Steele, I. H., Thrower, N., Noroian, P., \& Saleh, F. M. (2018). Understanding suicide across the lifespan: A United States perspective of suicide risk factors, assessment \& management. Journal of Forensic Sciences, 63(1), 162-171.

U.S. Department of Justice, Bureau of Justice Statistics. Survey of inmates in local jails, 2002: [United States] [Computer File]. Conducted by U.S. Department of Commerce, Bureau of the Census. Ann Arbor, MI: Inter-university Consortium for Political and Social Research [producer and distributor], 2006.

U.S. Department of Veterans Affairs. (2021). Eligibility. https://www.va.gov/health$\underline{\text { care/eligibility/ }}$

Weiner, J., Richmond, T. S., Conigliaro, J., \& Wiebe, D. J. (2011). Military veteran mortality following a survived suicide attempt. BMC Public Health, 11 (374), 1-9.

White, M. D., Mulvey, P., Fox, A. M., \& Choate, D. (2012). A hero's welcome? Exploring the prevalence and problems of military veterans in the arrestee population, Justice Quarterly, 29(2), 258-286, DOI:

$10.1080 / 07418825.2011 .560890$ 
Wood, D. S., Wood, B. M., Watson, A., Sheffield, D., \& Hauter, H. (2020). Veteran suicide risk factors: A national sample of nonveteran and veteran men who died by suicide. Health and Social Work, 45(1), 23-30.

Wortzel, H.S., Binswanger, I. A., Anderson, C. A., \& Adler, L. E. (2009). Suicide among incarcerated veterans. The Journal of the American Academy of Psychiatry and the Law, 37: 82-91. 